\title{
Tratamiento de agua residual procedente de lavadoras por el método de electrocoagulación para la reutilización en riego de vegetales - Ate Vitarte
}

\author{
Wastewater treatment from washing machines by the method of \\ electrocoagulation for the reuse in irrigation of vegetables - Ate Vitarte
}

\author{
María Vanessa Cuba Tello, Paul Alcocer Meneses ${ }^{2}$ Mariano Pacheco Ortíz ${ }^{3}$
}

Recibido: Junio 2019 - Aprobado: Julio 2019

\begin{abstract}
RESUMEN
La electrocoagulación $(E C)$ en los últimos años está llamando la atención para la eliminación de diferentes contaminantes presentes en diversos efluentes. A través de este trabajo, se verificó la viabilidad de utilizar la técnica de electrocoagulación como una alternativa ante los procesos químicos convencionales, en el tratamiento del agua residual de lavadoras para ser reutilizado en riego de vegetales. Analizadas regularmente. Se analizaron cuatro factores: intensidad, tiempo, distancia y $\mathrm{pH}$; con tres niveles para cada factor. Para optimizar el proceso de electrocoagulación sobre los factores de diseño, se realizaron experimentos de acuerdo al método de Taguchi. Se realizó el análisis de varianza (ANOVA) para ver el efecto de los variables sobre la remoción de la turbiedad y otros parámetros fisicoquímicos. Los resultados arrojaron que los parámetros de mayor influencia en la remoción de la turbiedad fueron tiempo e intensidad a comparación de los demás. La optimización de los factores sobre la alta remoción de los parámetros fisicoquímicos se obtuvo a los niveles de intensidad 5 Amperios, 15 minutos, 2 centímetros y pH 7.5, con una remoción de $99.02 \%$ turbiedad, $90.83 \%$ DQO y $95.93 \%$ SST, el efluente tratado se encuentra en el rango permisible para su uso en riego de vegetales.
\end{abstract}

Palabras clave: Agua gris; electrocoagulación (EC); electrodo; electrólisis; Taguchi.

\begin{abstract}
Electrocoagulation $(E C)$ in recent years is calling the growing attention for the elimination of different contaminants present in the various effluents. Through this work, the feasibility of using the electrocoagulation technique as an alternative to conventional chemical processes in the treatment of wastewater from washing machines to be reused in vegetable irrigation, analyzed regularly was verified. Four factors were analyzed: intensity, time, distance and $\mathrm{pH}$; with three levels for each factor. To optimize the electrocoagulation process on the design factors, the experiments were carried out according to the Taguchi method. The analysis of variance (ANOVA) was performed to see the effect of the variables on the removal of turbidity and other physicochemical parameters. The results showed that the parameters with the greatest influence on the removal of turbidity were time and intensity compared to the others. The optimization of the factors on the high removal of the physicochemical parameters was obtained at the levels of intensity 5 Amps, time of 15 minutes, distance of 2 centimeters and pH 7.5, with a removal of $99.02 \%$ in turbidity, $90.83 \%$ in COD Y $95.93 \%$ in SST, with which the treated effluent is in the permissible range for use in vegetable irrigation.
\end{abstract}

Keywords: Gray water; electrocoagulation (EC); electrode; electrolysis; Taguchi.

\footnotetext{
1 Unidad de Posgrado de la Facultad de Ingeniería Geológica, Minera, Metalúrgica y Geográfica de la Universidad Nacional Mayor de San Marcos, Av. Venezuela s/n cuadra 24, Lima - Perú. E-mail: vanecuba@gmail.com. Facultad de Ingenieria, Programa academico de Ingenieria Ambiental de la Universidad de Huanuco, carretera central Km 2.6, Huanuco - Perú. E-mail:mcuba@udh.edu.pe.

2 Universidad Nacional del Callao, Escuela de Posgrado de Ingeniería Química, Av. Juan Pablo II 306 Bellavista, Callao. E-mail:alcocermenesespaul@gmail.com. 3 Unidad de Posgrado de la Facultad de Ingeniería Geológica, Minera, Metalúrgica y Geográfica de la Universidad Nacional Mayor de San Marcos, Av. Venezuela s/n cuadra 24, Lima - Perú.
} 


\section{INTRODUCCIÓN}

Toda agua residual que proviene de todas las instalaciones domésticas menos del inodoro se conoce como aguas grises (GW), los cuales incluyen desecho de lavado de platos, baño, ducha y residuos de cocina. Estos representan un flujo importante en volumen por área por cada domicilio. La generación de aguas grises podría representar un 75\% en volumen de aguas residuales generadas por hogares (Leal, et al. 2011).

La composición del agua gris depende de algunas características tales como: cantidad de personas que viven en la casa, diferentes niveles de vida social y cultural, etc. Dichas aguas contienen surfactantes, concentraciones altas de jabones, así como alta concentración en materia orgánica. La composición promedio de las aguas grises en la literatura son: $\mathrm{pH}=6-9$, turbidez 12-2131 NTU, conductividad 1.4$703 \mathrm{mS} / \mathrm{m}$, sólidos suspendidos totales (TSS) 11-2180 mg / L, demanda bioquímica de oxígeno (DBO) 23-942 mg / L, demanda química de oxígeno (DQO) 55-2000 mg / L, principio activo de azul de metileno (MBAS) 0,3-118 $\mathrm{mg}$ / L, aceite y grasa (O\&G) 7-328 mg / L, coliformes totales (TC) 200-2.2 × 107MPN, formas de coliforme fecal (FC) 13-1.9 × 107MPN y Escherichia coli 10-3.9 × 105MPN (Ghaitidak \& Yadav, 2013)(Li et al. 2009).

El uso de lavadoras en estos tiempos es cada vez más creciente y el uso promedio de agua por lavado es de 42 a 62 litros de agua (lavadora con capacidad de carga de 7 kilos de ropa) (catálogo de equipos de lavandería industrial). El proceso químico de floculación se utilizó en una investigación para tratar aguas grises, con lo cual se redujo a $64 \%$ de DQO y $89 \%$ de DBO, dentro del estudio se observó que por sí sólo la floculación no puede eliminar la carga orgánica, por lo tanto se debe acoplar a otro proceso para obtener una calidad requerida para su reuso (Kariuki, et al. 2011).

Los sistemas de tratamiento para efluentes relacionados con la electroquímica son muy prometedores, dentro de ellos tenemos a la electrocoagulación (EC), la electrooxidación y la electroflotación, hasta la actualidad estos métodos son utilizados para eliminar eficazmente diversos contaminantes (Barısc1, et al. 2015), (Ulu, et al. 2014). La electrocoagulación es un método alternativo, fácil y efectivo para tratar todo tipo de efluente líquido, las reacciones que suceden tanto en el ánodo y cátodo son oxidación y reducción respectivamente, cuando se hace pasar corriente continua sobre los electrodos se generan iones de aluminio y hierro, con diferentes grados de oxidación, los cuales en medios acuosos forman hidróxidos, quienes actúan como desestabilizadores de las partículas presentes en los líquidos, el mecanismo de eliminación puede ser como adsorción, neutralización y coagulación. Posibles reacciones químicas en una unidad de proceso por lotes de reactor de electrocoagulación se presentan en la Figura 1. Durante el proceso de electrocoagulación, la corriente aplicada promueve reacciones de electrodisoluciones, generando cationes metálicos ehidróxidos de metal en la solución(Vepsäläinen, et al. 2012). Este método de electrocoagulación ofrece varias ventajas con respecto al método tradicional de coagulación química, ofreciendo costo y efectividad, compatibilidad, no se necesita agregar otro producto químico al tratamiento. El electrodo de mayor uso por su amplia característica y ventajas son el aluminio y hierro, los cuales producen hidróxidos. Hay estudios realizados sobre su uso como electrodos de aluminio y hierro para tratar aguas residuales provenientes de la industria láctea, donde se obtuvo un 79.2\% de eliminación de DQO se informó en (Yavuz, et al. 2011). Hay poca información sobre el uso de electrocoagulación para el tratamiento de aguas grises, autores como Vakil, et al. Investigaron utilizando electrodos de aluminio con lo cual redujeron de $380 \mathrm{mg} / \mathrm{L}$ a $160 \mathrm{mg} / \mathrm{L}$ el valor de COD, la turbidez se redujo de 104 NTU a 15.6 NTU, también hubo una significancia en la eliminación de coliformes (Vakil, et al. 2014). En otros estudios se usaron electrodos de aluminio para tratamiento de aguas grises, en los cuales arrojaron valores de DQO, turbidez y coliformes totales en $463 \mathrm{mg} / \mathrm{L}, 133$ NTU y $78 \mathrm{mg} / \mathrm{L}$ respectivamente (Bani-

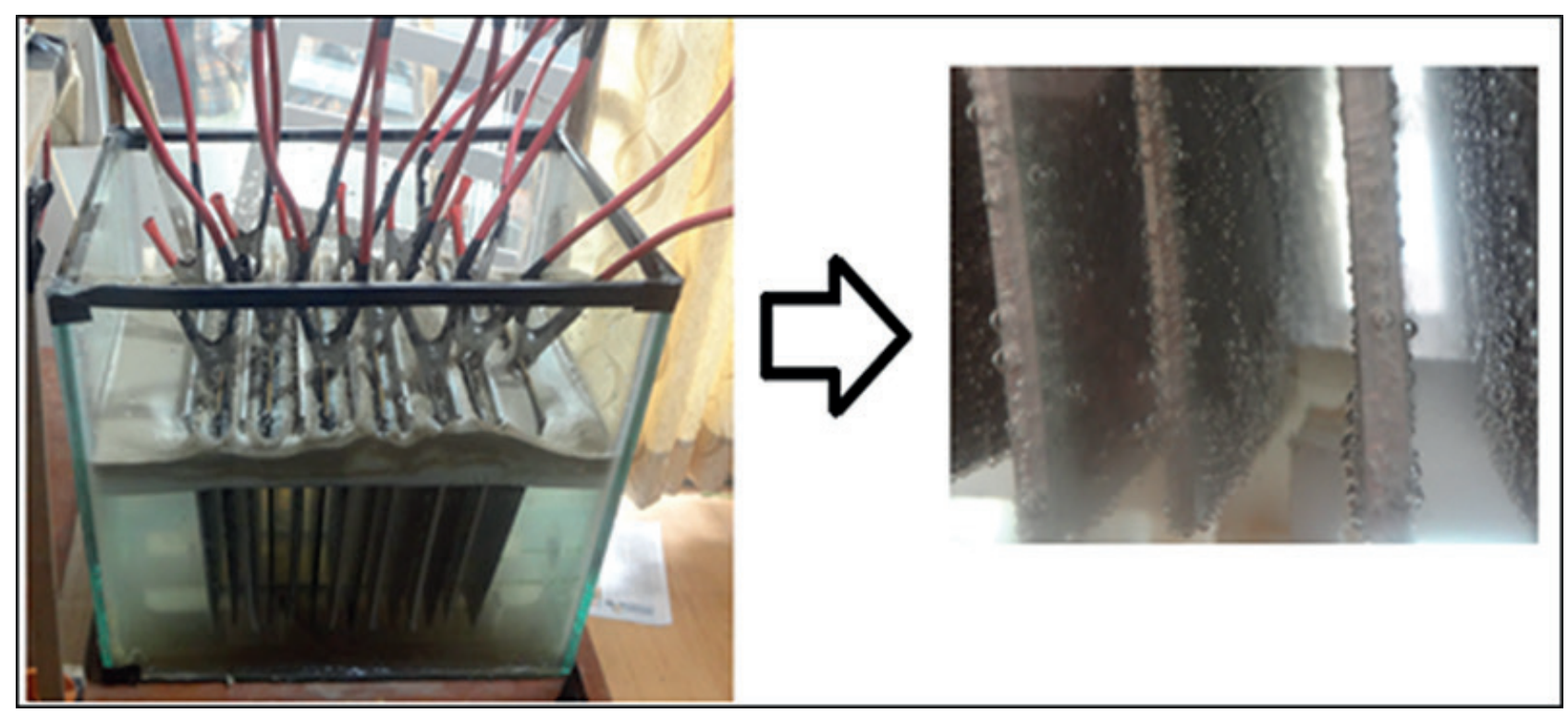

Figura 1. Equipo de electrocoagulación donde se libera los Gases de hidrogeno y oxígeno 
Melhem \& Smith, 2012). En otro estudio se utilizaron la electrocoagulación para tratar el efluente de aguas grises, evaluando las diferentes configuraciones de electrodos entre aluminio y hierro, obteniendo la máxima eficiencia en la eliminación de COD con la configuración de Al-FeFe-Al, con densidad de corriente en $1 \mathrm{~mA} / \mathrm{cm}^{2}$ a $\mathrm{pH}$ de 7.62 (Barısc1 \& Turkay, 2016).

En este estudio, se analizó el efluente proveniente de lavadoras domésticas por medio de la electrocoagulación para su posterior reuso en el riego de vegetales, donde se utilizó electrodos de aluminio y acero inoxidable, no existen estudios publicados sobre el uso de estos dos tipos de electrodos hasta la actualidad para el tratamiento de efluentes de lavadoras. Se estudiaron las condiciones óptimas de operación tales como la intensidad, tiempo, distancia y $\mathrm{pH}$ para la eliminación de la turbiedad, DQO y DBO. Los ensayos experimentales se basaron por el método de arreglo ortogonal de Taguchi, otro de los objetivos también es determinar las condiciones óptimas para la máxima eliminación de los contaminantes en el efluente, y llegar a los parámetros establecidos para su uso en riego de vegetales según las normas establecidas.

En la investigación se realizó utilizando la metodología Taguchi, en optimizar los parámetros operacionales como intensidad, tiempo, distancia y $\mathrm{pH}$ en función de la respuesta. También podemos observar que en otros procesos se utilizaron el método de Taguchi tal como los siguientes trabajos de investigaciones de Tratamiento de vinazas por electrocoagulación-electroflotación utilizando el método Taguchi, Recuperación de metales pesados de aguas residuales industriales utilizando el método Taguchi y Optimización del consumo de energía en la eliminación electrostática de arsénico del agua subterránea por el método Taguchi. En el trabajo de investigación se utilizaron electrodos (aluminio-acero inoxidable), aplicados sobre este tipo de aguas residuales, lo novedoso como tecnología alternativa emergente es aplicable para el reusó de riego de vegetales (Figura 2).

\section{MÉTODOS}

Mediante esta metodología experimental se controla deliberadamente las variables para delimitar relaciones entre ellas y está basado en la metodología científica. Esta investigación se enmarca en la parte experimental de tipo correlacional, descriptivo y analítico, basado de acuerdo a las hipótesis:

1. Caracterizando cualitativamente el efluente, se podrá establecer los factores de operación.

2. Optimizando los parámetros adecuados, se obtendrá un efluente apto para riego.

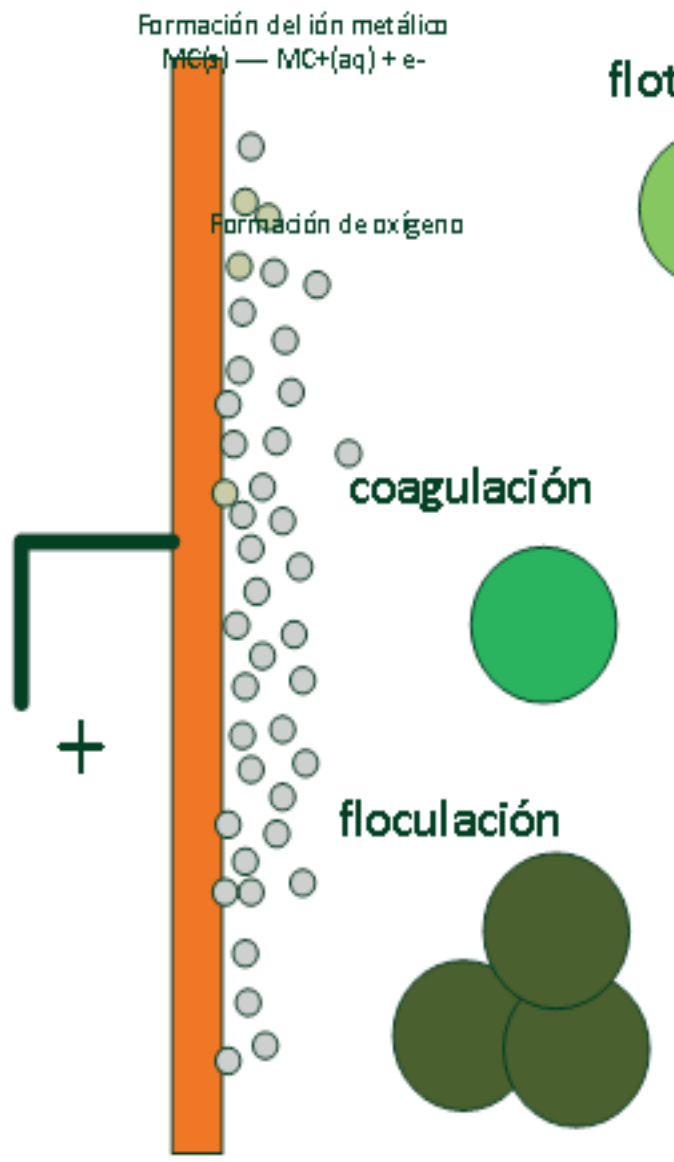

Electrodo ánodo

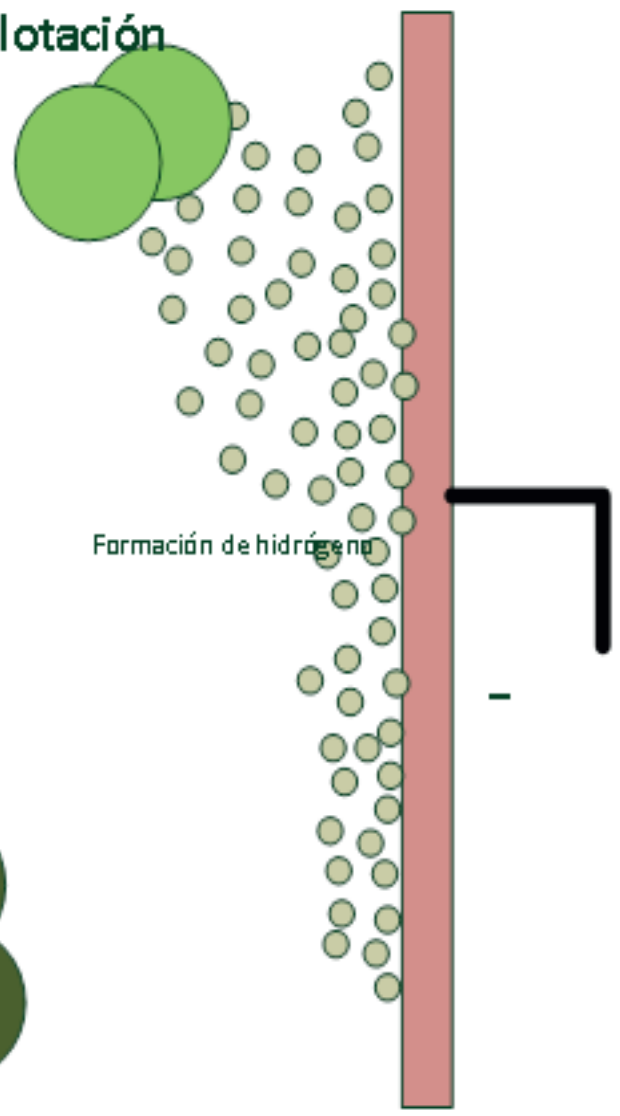

Electrodo cátodo

Figura 2. Vista esquemática de una celda electroquímica (CE). 
3. Determinando la eficiencia del módulo de electrocoagulación, se podrá conocer la eliminación en mayor porcentaje del contaminante) y los objetivos planteados.

La aplicación de la metodología para esta investigación está determinada en hacer una serie de experimentos ajustados en un diseño experimental para la optimización de la eficiencia en la eliminación de contaminantes sujetos al objetivo general planteado en el trabajo de investigación "Tratamiento de agua residual procedente de lavadoras por el método de electrocoagulación para la reutilización en riego de vegetales - Ate Vitarte" y poder facilitar como información precedente para posteriores trabajos de investigación.

En la Figura 3, se muestra un esquema corto de flujograma del trabajo realizado, donde se muestran las partes del montaje experimental de la electrocoagulación.

\subsection{Materiales}

El efluente doméstico provenientes de lavadoras se recogió del condominio Jardines de Santa Clara Ate - Vitarte, se trabajó con un volumen total de 200 litros para toda la corrida, incluido las réplicas, se dividieron en tres lotes con la finalidad de acondicionar los parámetros establecidos para cada corrida, la primera solución se deja al mismo pH 7.5, la segunda se baja el pH a 5, utilizando ácido clohídrico $(\mathrm{HCl})$ y el tercero se sube el $\mathrm{pH}$ a 10 con soda caustica $(\mathrm{NaOH})$, luego se almacenaron las tres muestras a $4^{\circ} \mathrm{C}$ para su posterior estudio. Como electrodos se utilizaron en el ánodo el aluminio y en el cátodo el acero inoxidable, como electrodo de sacrificio se utilizó el aluminio. En la Tabla 1 se presenta la caracterización del efluente antes de ser tratado.

\subsection{Montaje experimental}

El tratamiento se llevó en lotes tipo bach, sobre un sistema construido a base de vidrio de forma rectangular con una capacidad de 15 litros. La configuración de los electrodos fue de tipo monopolar en paralelo, separados sobre tres niveles de configuración según el diseño propuesto, las dimensiones de los cátodos y ánodos eran $200 \times 175 \times 3 \mathrm{~mm}$ y 200x175x3 mm respectivamente, con el área de contacto $700 \mathrm{~cm}^{2}$ por electrodo. El proceso de electrocoagulación se llevó en funcionamiento gracias a una fuente de corriente continua.

Tabla 1. Caracterización del efluente antes de ser tratado

\begin{tabular}{lcc}
\hline $\begin{array}{l}\text { Parámetro Fisicoquímico y } \\
\text { Orgánico }\end{array}$ & Unidades & Resultado \\
Aceites y grasas & $\mathrm{mg} / \mathrm{L}$ & 21.61 \\
Conductividad & $\mu \mathrm{S} / \mathrm{cm}$ & 12.51 \\
Demanda química de oxígeno & $\mathrm{mg} / \mathrm{L}$ & 109 \\
Demanda biológica de oxígeno & $\mathrm{m} / \mathrm{L}$ & 286 \\
Detergentes & $\mathrm{SAAM}$ & 7.2636 \\
Sulfatos & $\mathrm{mg} / \mathrm{L}$ & 297 \\
Temperatura & ${ }^{\circ} \mathrm{C}$ & 24 \\
Turbiedad & $\mathrm{NTU}$ & 85.5 \\
Sólidos suspendidos totales & $\mathrm{mg} / \mathrm{L}$ & 615 \\
$\mathrm{pH}$ & - & 7.5 \\
\hline
\end{tabular}

Fuente: Resultados analizados por la Empresa Typsa.Perú. Metodología Experimental

Para poder analizar sistemáticamente el proceso de tratamiento por electrocoagulación, se basó en la metodología de Taguchi, con la finalidad de realizar

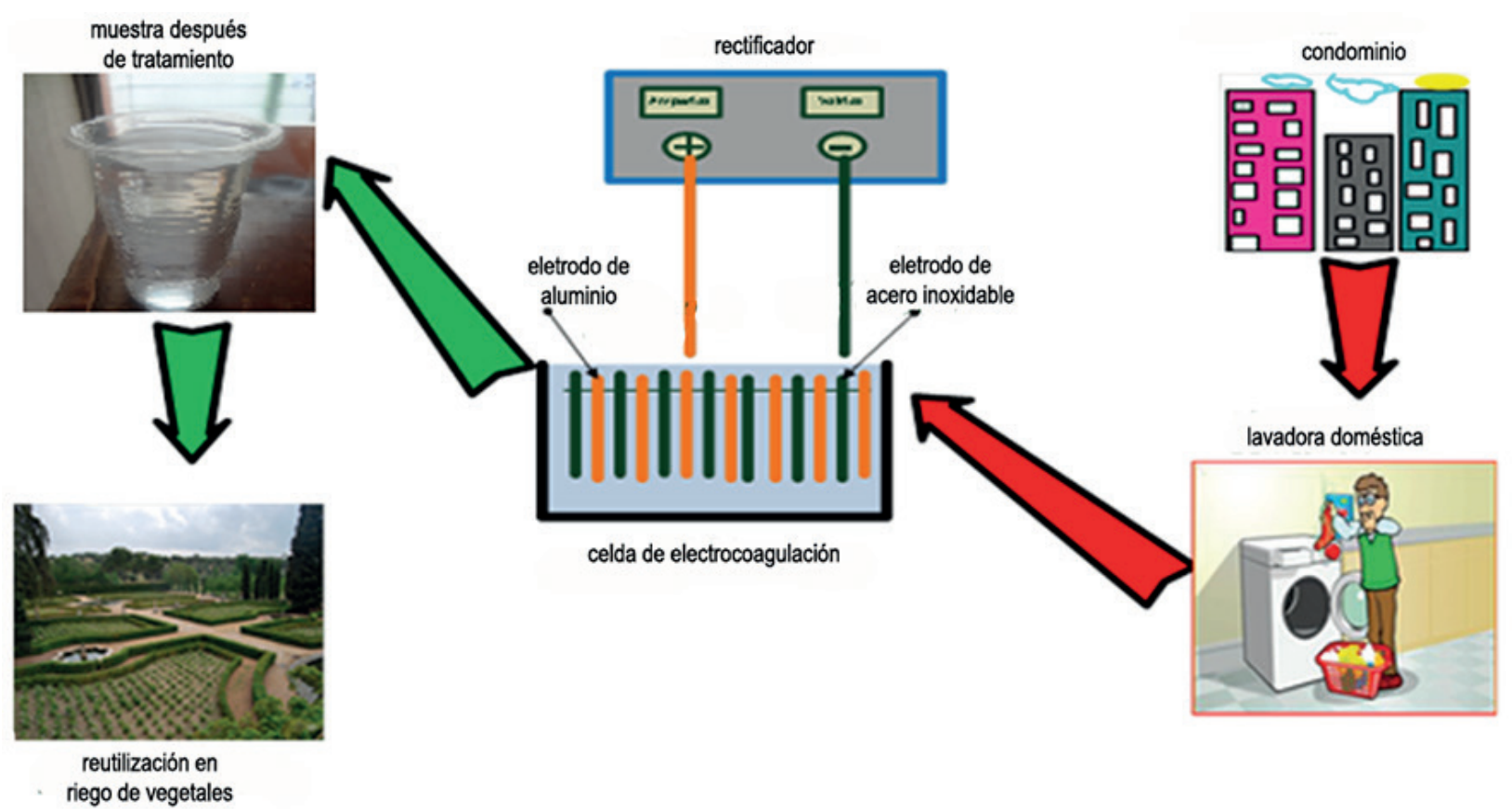

Figura 3. Esquema de un flujograma del trabajo de investigación. 
mínimas corridas para optimizar los factores en relación a una buena eficiencia de eliminación de contaminantes. Se consideraron cuatro factores que incluyen el tiempo (minutos), la intensidad (Amperios), distancia ( $\mathrm{mm}$ ) y por último el $\mathrm{pH}$, que han sido considerados como variables de control y la eficiencia en la eliminación de la turbiedad (NTU), demanda química de oxígeno y sólidos suspendidos totales $(\mathrm{ml} / \mathrm{L})$, como variables de señales. Cada factor ha sido analizado en tres niveles, en total según el diseño ortogal de Taguchi se consideran 9 experimentos con una réplica para cada corrida con la condición de que los datos sean fiables. La variable respuesta es el porcentaje de eliminación de turbiedad (NTU), sólidos suspendidos totales $(\mathrm{ml} / \mathrm{L})$ y demanda química de oxígeno $(\mathrm{ml} / \mathrm{L})$. En la Tabla 2 se muestra en forma resumida los factores de control y señal con sus respectivos niveles.

\subsubsection{Procedimiento Experimental}

Antes de empezar con las corridas, los electrodos fueron lijados con lija fina y sumergidos en una solución de ácido clorhídrico de baja concentración $(0.1 \%)$ y otra solución de soda cáustica $(0.05 \mathrm{M})$, para eliminar los restos pegados en la superficie de los electrodos, finalmente son lavados con agua destilada para su utilización. Se trabajó en base de 10 litros de efluente por cada corrida, se dividieron en tres grupos el efluente a tratar, porque se trabajó en tres niveles de $\mathrm{pH}$, las muestras tomadas antes y después de ser tratados se enviaron a un laboratorio garantizado. Antes de cada corrida los electrodos se lijaron y se sumergieron en una solución de $\mathrm{HCl}(0.1 \%)$ en peso, y después se lavaron con agua destilada, todo estos pasos se repitieron para cada corrida. La eficiencia para cada corrida se evaluó conociendo las concentraciones iniciales y finales de la muestra, antes de ser analizadas las muestras se filtraron con papel filtro número $0.45 \mathrm{um}$, el porcentaje de eliminación de los factores de señal se calculó como sigue:

Eliminación de la turbiedad $(\%)=\frac{(\text { Turbiedad }(e)-\text { Turbiedad }(s))}{\text { Turbiedad }(s)} \times 100$

\section{$\underline{\text { Análisis Estadístico }}$}

El análisis de varianza (ANOVA) se determinó utilizando el Software Minitab 17. El ANOVA realiza la influencia de los parámetros de control mediante la evaluación de la variación de los resultados causados por cada factor de control sobre los factores de respuesta, que también pueden incluir factores de ruido (Roy, 2001).

\section{RESULTADOS}

Una vez terminado las corridas, se enviaron las muestras conservadas a un laboratorio certificado para su respectivo análisis de sus parámetros, en este caso los resultados se muestran en la Tabla 3, donde se comparan de los 9 corridas después de su tratamiento (Figura 4, Tabla 4).

En las Tablas 3 y 4 , se observa los resultados obtenidos en el laboratorio después de su análisis instrumental, donde

Tabla 2. Descripción de experimentos por la metodología Taguchi con sus respectivos niveles de cada factor

\begin{tabular}{cccccc}
\hline Prueba experimental & Intensidad (X1) (Amperios) & $\mathrm{pH} \mathrm{(X2)}$ & Tiempo (X3) (Minutos) & Distancia (X4) (Centímetros) & Respuesta (Y) \\
\hline 1 & 3 & 5 & 10 & 1 & $\mathrm{Y}$ \\
2 & 3 & 7.5 & 15 & 1.5 & $\mathrm{Y}$ \\
3 & 3 & 10 & 20 & 2 & $\mathrm{Y}$ \\
4 & 5 & 5 & 15 & 2 & $\mathrm{Y}$ \\
5 & 5 & 7.5 & 20 & 1 & $\mathrm{Y}$ \\
6 & 5 & 10 & 10 & 1.5 & $\mathrm{Y}$ \\
7 & 8 & 5 & 20 & 2 & $\mathrm{Y}$ \\
8 & 8 & 7.5 & 10 & 1 & $\mathrm{Y}$ \\
9 & 8 & 10 & 15 & & \\
\hline
\end{tabular}

Fuente: Esquema realizado en el Software Minitab utilizando la Metodología Taguchi.

Tabla 3. Los resultado de la remoción de la demanda química de oxígeno, turbiedad y solidos totales suspendidos para cada experimentos

\begin{tabular}{|c|c|c|c|c|c|c|}
\hline Experimentos & $\begin{array}{l}\text { Demanda química } \\
\text { de oxígeno }(\mathrm{mL} / \mathrm{L})\end{array}$ & Turbiedad (NTU) & $\begin{array}{l}\text { Sólidos suspendidos } \\
\text { totales (mL/L) }\end{array}$ & $\begin{array}{c}\text { \% Remoción de } \\
\text { DQO }\end{array}$ & $\begin{array}{l}\text { \% Remoción de } \\
\text { Turbiedad }\end{array}$ & \% Remoción de SST \\
\hline 1 & 71 & 62 & 198 & 34.86 & 27.49 & 67.8 \\
\hline 2 & 47 & 28 & 97 & 56.88 & 67.25 & 84.23 \\
\hline 3 & 35 & 15 & 61 & 67.89 & 82.46 & 90.08 \\
\hline 4 & 60 & 23 & 83 & 44.95 & 73.1 & 86.5 \\
\hline 5 & 10 & 0.84 & 25 & 90.83 & 99.02 & 95.93 \\
\hline 6 & 79 & 57 & 142 & 27.52 & 33.33 & 76.91 \\
\hline 7 & 18 & 10.5 & 46 & 83.49 & 87.72 & 92.52 \\
\hline 8 & 78 & 53.9 & 153 & 28.44 & 36.96 & 75.12 \\
\hline 9 & 41 & 13.5 & 50 & 62.39 & 84.21 & 91.87 \\
\hline
\end{tabular}




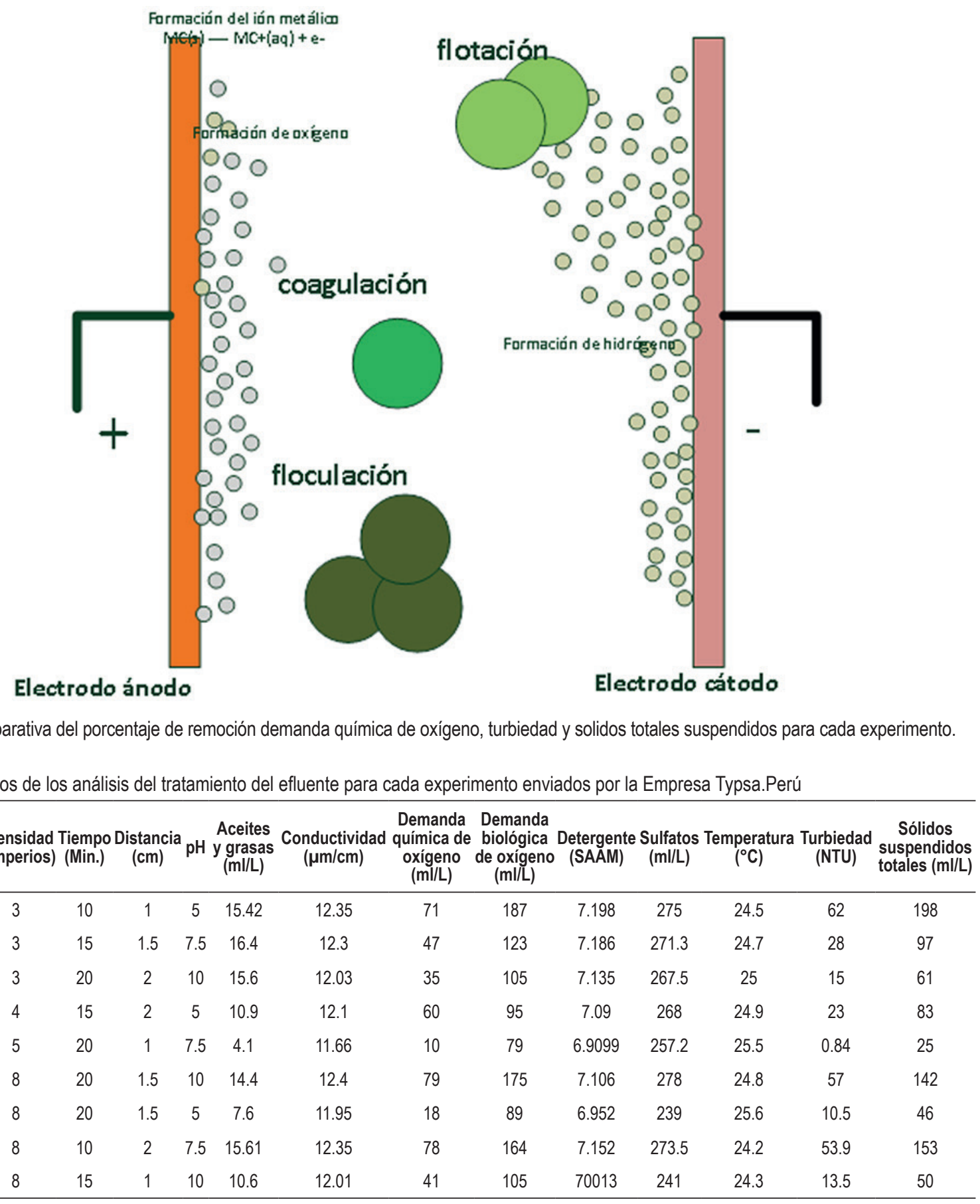

Figura 4. Comparativa del porcentaje de remoción demanda química de oxígeno, turbiedad y solidos totales suspendidos para cada experimento.

Tabla 4. Resultados de los análisis del tratamiento del efluente para cada experimento enviados por la Empresa Typsa.Perú

\begin{tabular}{|c|c|c|c|c|c|c|c|c|c|c|c|c|c|}
\hline Experimentos & $\begin{array}{l}\text { Intensidad } \\
\text { (Amperios) }\end{array}$ & $\begin{array}{l}\text { Tiempo } \\
\text { (Min.) }\end{array}$ & $\begin{array}{l}\text { Distancia } \\
\text { (cm) }\end{array}$ & $\mathrm{aH}$ & $\begin{array}{l}\text { Aceites } \\
\text { y grasas } \\
\text { (ml/L) }\end{array}$ & $\begin{array}{c}\text { Conductividad } \\
(\mu \mathrm{m} / \mathrm{cm})\end{array}$ & $\begin{array}{l}\text { Demanda } \\
\text { química de } \\
\text { oxígeno } \\
\text { (ml/L) }\end{array}$ & $\begin{array}{l}\text { Demanda } \\
\text { biológica } \\
\text { de oxígeno } \\
(\mathrm{ml} / \mathrm{L})\end{array}$ & $\begin{array}{c}\text { Detergente } \\
\text { (SAAM) }\end{array}$ & $\begin{array}{l}\text { Sulfatos } \\
(\mathrm{ml} / \mathrm{L})\end{array}$ & Temperatura & $\begin{array}{l}\text { Turbiedad } \\
\text { (NTU) }\end{array}$ & $\begin{array}{c}\text { Sólidos } \\
\text { suspendidos } \\
\text { totales (ml/L) }\end{array}$ \\
\hline Exterimento 01 & 3 & 10 & 1 & 5 & 15.42 & 12.35 & 71 & 187 & 7.198 & 275 & 24.5 & 62 & 198 \\
\hline Exterimento 02 & 3 & 15 & 1.5 & 7.5 & 16.4 & 12.3 & 47 & 123 & 7.186 & 271.3 & 24.7 & 28 & 97 \\
\hline Exterimento 03 & 3 & 20 & 2 & 10 & 15.6 & 12.03 & 35 & 105 & 7.135 & 267.5 & 25 & 15 & 61 \\
\hline Exterimento 04 & 4 & 15 & 2 & 5 & 10.9 & 12.1 & 60 & 95 & 7.09 & 268 & 24.9 & 23 & 83 \\
\hline Exterimento 05 & 5 & 20 & 1 & 7.5 & 4.1 & 11.66 & 10 & 79 & 6.9099 & 257.2 & 25.5 & 0.84 & 25 \\
\hline Exterimento 06 & 8 & 20 & 1.5 & 10 & 14.4 & 12.4 & 79 & 175 & 7.106 & 278 & 24.8 & 57 & 142 \\
\hline Exterimento 07 & 8 & 20 & 1.5 & 5 & 7.6 & 11.95 & 18 & 89 & 6.952 & 239 & 25.6 & 10.5 & 46 \\
\hline Exterimento 08 & 8 & 10 & 2 & 7.5 & 15.61 & 12.35 & 78 & 164 & 7.152 & 273.5 & 24.2 & 53.9 & 153 \\
\hline Exterimento 09 & 8 & 15 & 1 & 10 & 10.6 & 12.01 & 41 & 105 & 70013 & 241 & 24.3 & 13.5 & 50 \\
\hline
\end{tabular}

Fuente: Resultados realizados con los equipos para monitoreo de la calidad del agua y por la empresa Typsa Perú.

se aprecia la remoción considerada de la turbidez, sólidos suspendidos totales y demanda química de oxígeno, el experimento de mayor aceptación sobre nuestros objetivos:

1. Caracterizar cualitativamente a las aguas residuales de la lavadora.

2. Los principales parámetros operacionales más adecuados.

3. Determinar la eficiencia del módulo de electrocoagulación.

4. Caracterizar cuantitativamente los componentes del efluente tratado) fue la corrida 5 , con niveles de 5 de Amperios, en el tiempo 15 minutos, distancia de 2 centímetros y a $\mathrm{pH} 7.5$, logrando así bajar la turbiedad a $0.84 \mathrm{NTU}, 10 \mathrm{ml} / \mathrm{L}$ de demanda química de oxígeno y llegar a $25 \mathrm{ml} / \mathrm{L}$ de sólidos suspendidos totales, concluyendo la mayor aceptación para la reutilización en el riego de vegetales.

En la Tabla 5, de respuesta para medias de turbiedad, solidos totales suspendidos, demanda química de oxígeno en relación con las 4 variables, se identifica que el factor que tiene mayor efecto es el Tiempo, el cual tiene una clasificación de 1, seguidamente de la Intensidad, 


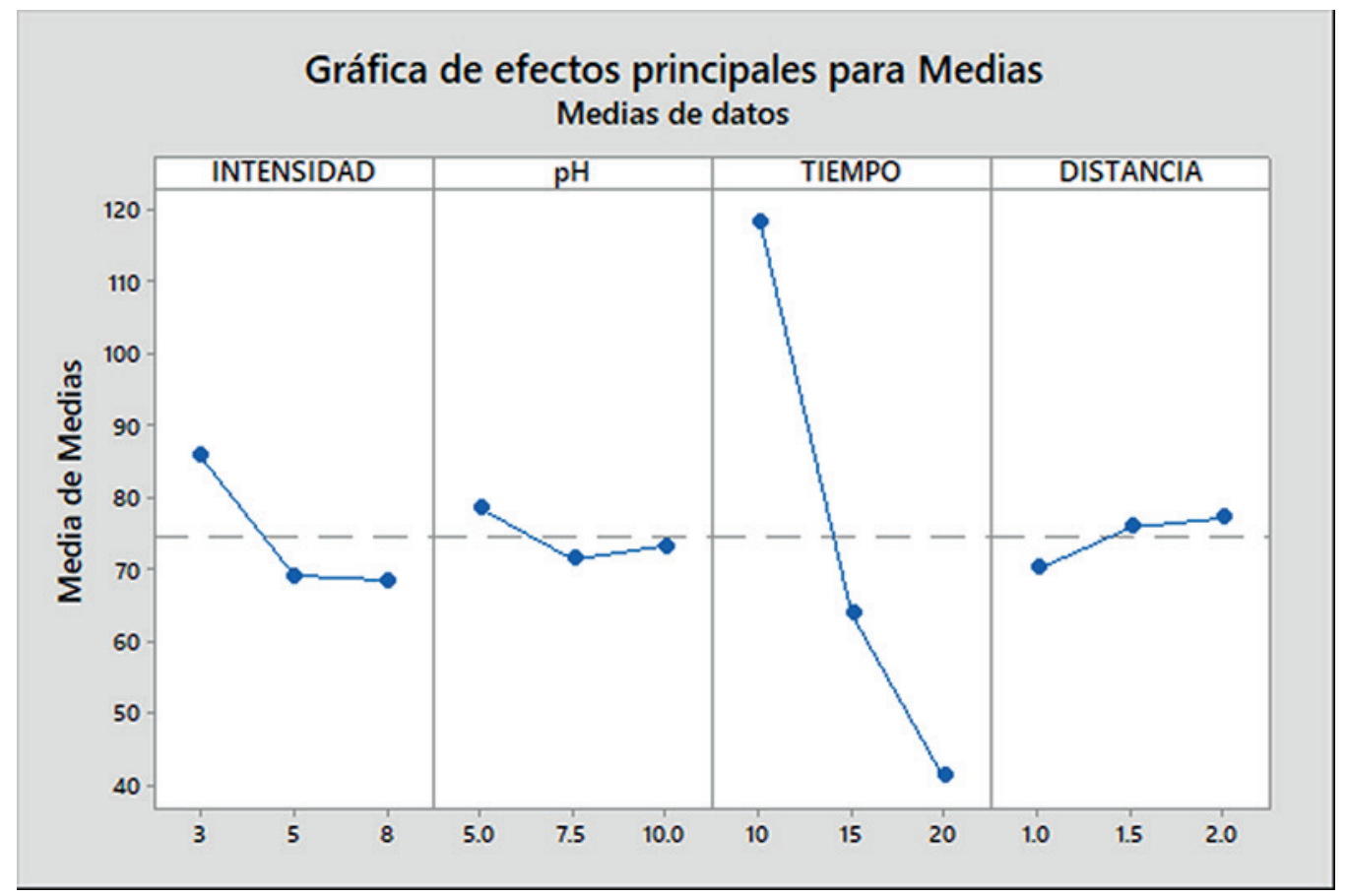

Figura 5. Los efectos principales para medias de las variables de intensidad, $\mathrm{pH}$, tiempo y distancia. Resultados de las medias de las variables utilizando el Software de Minitad.

$\mathrm{pH}$ y distancia con rangos de clasificación de 2, 3 y 4 respectivamente. Asimismo, para el factor Tiempo, en el nivel 1 el promedio máximo para las medias es 118.33 y el promedio en el nivel 3 es 41.20.

En la Figura 5, se observa los efectos principales para la medias de la variable de turbiedad, y tiempo, donde se tiene el mayor efecto en relación a los demás factores, Además, el tiempo promedio de $10 \mathrm{~min}$. Tiene una relación más alta con la turbiedad; seguidamente de una intensidad de 3 amperios, a diferencia de la distancia y el $\mathrm{pH}$ que tienen un menor efecto.

Tabla 5. Tabla de las medias de los parámetros de turbiedad, solidos totales suspendidos, demanda química de oxígeno en relación con las variables

\begin{tabular}{ccccc}
\hline Nivel & Intensidad & pH & Tiempo & Distancia \\
\hline 1 & 85.75 & 78.54 & 118.33 & 70.2 \\
2 & 69.07 & 71.56 & 63.79 & 75.96 \\
3 & 68.49 & 73.21 & 41.2 & 77.16 \\
Delta & 17.26 & 6.98 & 77.13 & 6.96 \\
Clasificar & 2 & 3 & 1 & 4 \\
\hline
\end{tabular}

Fuente: Resultados de las medias de los parámetros (turbiedad solidos totales y suspendidos y demanda química de oxigeno) obtenidas realizando el análisis de ANOVA.

De la Tabla 6, tabla ANOVA, muestra que los parámetros: Intensidad, $\mathrm{pH}$, Tiempo y Distancia respecto a la Turbiedad, los valores del p-valor (significativo) se encuentra en el parámetro Tiempo ( $\mathrm{p}$-valor $=0.003$ ) el cual tiene una contribución del $87.30 \%$ sobre la explicación de respuesta, en comparación a los otros factores que nos son significativos. Asimismo, este resultado confirma lo realizado en el diseño experimental de Taguchi (ver Figura 5), el cual identificó el factor que tiene mayor efecto es el Tiempo.

El resultado según la Tabla 6; se observa que el tiempo tiene una relación directa con la optimización de los parámetros del módulo de electrocoagulación, esto quiere decir que cuanto más tiempo paso el proceso hay una mayor optimización en la eliminación de los parámetros físico químicos y biológicos en los resultados con respecto al reusó del agua para riego de los vegetales. Donde también podemos decir en la hipótesis que la eficiencia va incrementándose cuanto más tiempo transcurre el proceso.

Tabla 6. Tabla ANOVA. Turbiedad respecto a la intensidad, pH, tiempo y distancia

\begin{tabular}{lccccc}
\hline Factores & DF & SS & Contribución & F-valor & p-valor \\
\hline Intensidad & 1 & 108.5 & $2.65 \%$ & 1.22 & 0.331 \\
pH & 1 & 16.67 & $0.41 \%$ & 0.19 & 0.687 \\
Tiempo & 1 & 3580 & $87.30 \%$ & 40.32 & 0.003 \\
Distancia & 1 & 40.35 & $0.98 \%$ & 0.45 & 0.537 \\
Error & 4 & 355.2 & $8.66 \%$ & & \\
\hline
\end{tabular}

Fuente: Resultados del análisis de ANOVA de las variables de intensidad, pH, tiempo y distancia.

De la Tabla 7, Tabla ANOVA, muestra los parámetros: Intensidad, $\mathrm{pH}$, Tiempo y Distancia respecto a la a la Demanda química, los valores del p-valor (significativo) se encuentran en los parámetros: Tiempo $(\mathrm{p}$-valor $=0.000)$ y Distancia ( $\mathrm{p}$-valor $=0.008)$; los cuales presentan una contribución del $89.12 \%$ y $8.51 \%$ respectivamente sobre 
la explicación de respuesta, en comparación a los otros factores que nos son significativos. También, este resultado confirma lo realizado en el diseño experimental de Taguchi (ver Figura 5), el cual identificó los factores que tienen mayor efecto es el Tiempo y Distancia.

Tabla 7. Tabla ANOVA. Demanda química respecto a la intensidad, $\mathrm{pH}$, tiempo y distancia

\begin{tabular}{lccccc}
\hline Factores & DF & SS & Contribución & F-valor & p-valor \\
\hline Intensidad & 1 & 44.96 & $0.88 \%$ & 2.58 & 0.183 \\
$\mathrm{pH}$ & 1 & 6 & $0.12 \%$ & 0.34 & 0.589 \\
Tiempo & 1 & 4538 & $89.12 \%$ & 260.79 & 0 \\
Distancia & 1 & 433.5 & $8.51 \%$ & 24.92 & 0.008 \\
Error & 4 & 69.6 & $1.37 \%$ & & \\
\hline
\end{tabular}

Fuente: Elaborado utilizando el software de Minitad

De la Tabla 8, Tabla ANOVA, muestra los parámetros: Intensidad, $\mathrm{pH}$, Tiempo y Distancia respecto a los Solidos suspendidos, el valor del p-valor (significativo) se encuentra en el parámetro Tiempo ( $\mathrm{p}$-valor=0.004); el cual presenta una contribución del $81.01 \%$ sobre la explicación de respuesta, en comparación a los otros factores que nos son significativos. Asimismo, este resultado confirma lo realizado en el diseño experimental de Taguchi (ver Figura 5), el cual identificó el factor que tiene mayor efecto es el Tiempo.

Tabla 8. Tabla ANOVA. Residuos suspendidos respecto a la intensidad, $\mathrm{pH}$, tiempo y distancia

\begin{tabular}{lccccc}
\hline Factores & DF & SS & Contribución & F-valor & p-valor \\
\hline Intensidad & 1 & 1645 & $6.13 \%$ & 2.7 & 0.176 \\
$\mathrm{pH}$ & 1 & 912.7 & $3.40 \%$ & 1.5 & 0.288 \\
Tiempo & 1 & 21720 & $81.01 \%$ & 35.63 & 0.004 \\
Distancia & 1 & 96 & $0.36 \%$ & 0.16 & 0.712 \\
Error & 4 & 2438 & $9.09 \%$ & & \\
\hline
\end{tabular}

Fuente: Resultados del análisis de ANOVA de las variables

La intensidad de corriente es uno de los factores de mayor importancia para controlar el buen rendimiento de la celda. La densidad de corriente aplicada determina la tasa de liberación de coagulante, la producción de burbujas y el crecimiento de flóculos (Kumar \& Goel, 2010). La intensidad según los resultados arrojados dan un efecto importante sobre la reducción de la demanda química de oxígeno, la turbiedad y sólidos suspendidos totales, el nivel con que dio mayores resultados fue de 8 Amperios.

El efecto del $\mathrm{pH}$ inicial es otro parámetro importante que afecta la eficiencia de proceso de la CE (Irdemez, et al.2006). Para apreciar la influencia del $\mathrm{pH}$ sobre el porcentaje de remoción de la turbiedad se trabajó a tres niveles de los cuales el pH de mayor influencia se dio en 7.5.

\section{CONCLUSIONES}

- Se evaluó en las condiciones de ácido, neutro y básico: donde se encontró la mejor reducción de los contaminantes fue a la condición de $\mathrm{pH}$ $=7,5$; dando un porcentaje de remoción de los contaminantes de DQO de $90.83 \%$, Turbiedad de
99.02 y Solidos Totales Suspendidos de 95.93\% Sobre las aguas residuales provenientes de lavadoras se caracterizaron los parámetros como $\mathrm{pH}$, color, temperatura, conductividad eléctrica, aceite y grasas, etc

- Se encontraron los niveles óptimos ( $\mathrm{pH}=7.5$, intensidad $=8$ amperios, distancia $=1 \mathrm{~cm}$ y tiempo $=20$ minutos) para cada factor dando resultados positivos en el tratamiento, logrando reducir el DQO $=90.83 \%$, turbiedad $=99.02 \%$ y sólidos suspendidos totales $=94.93 \%$ ) por ende los niveles más significativos de tiempo es a los 20 minutos, intensidad de 8 Amperios, distancia a 2 centímetros y por último a nivel de $\mathrm{pH} 7.5$, logrando en la eliminación de la turbiedad, demanda química de oxígeno y sólidos suspendidos totales.

- Al corroborar la eficiencia del módulo sobre la eliminación de contaminantes ha sido positivo con respecto a la turbiedad, demanda química del oxígeno y sólidos suspendidos totales, como se observa en la gráfica 1.

- Las medias para turbiedad, solidos totales suspendidos, demanda química de oxígeno en relación con las 4 variables, se identifica que el factor que tiene mayor efecto es el Tiempo, el cual tiene una clasificación de 1, seguidamente de la Intensidad, $\mathrm{pH}$ y Distancia con rangos de clasificación de 2, 3 y 4 respectivamente. Asimismo, para el factor Tiempo, en el nivel 1 el promedio máximo para las medias es 118.33 y el promedio en el nivel 3 es 41.20 .

\section{AGRADECIMIENTOS}

Agradecer a las personas e instituciones que hicieron posible el desarrollo de la investigación proporcionando la información necesaria para el análisis y procesamiento; y a la Revista del Instituto de Investigación de la Facultad de Ingeniería Geológica, Minera, Metalúrgica y Geográfica de la Universidad Nacional Mayor de San Marcos.

\section{REFERENCIAS BIBLIOGRÁFICAS}

Aguilar, E. (2015). Evaluación de la eficiencia de una celda de electrocoagulación a escala laboratorio para el tratamiento de agua. Revista Del Instituto De Investigación De La Facultad De Ingeniería Geológica, Minera, Metalúrgica Y Geográfica, 18(35), pp. 69-73. Recuperado de https:// revistasinvestigacion.unmsm.edu.pe/index.php/iigeo/ article/view/11843

Bani-Melhem, K., \& Smith, E. (2012). Grey water treatment by a continuous process of an electrocoagulation unit and a submerged membrane bioreactor system. Chemical Engineering Journal 198 - 199, 201-210. DOI: https://doi. org/10.1016/j.cej.2012.05.065

Barısc1, S., Sarkka, H., Sillanpaa, M., \& Dimoglo, A. (2015). The treatment of greywater from a restaurant by electrosynthesized ferrate. Desalination and Water Treatment, 1-11. DOI:https:// doi.org/10.1080/19443994.2015.1038740 
Barısc1, S., \& Turkay, O. (2016). Domestic greywater treatment by electrocoagulation using hybrid electrode combinations. Journal of Water Process Engineering 10, 56-66. DOI: https://doi.org/10.1016/j.jwpe.2016.01.015

Bermeo G., M., \& Tinoco G., Óscar. (2017). Remoción de colorantes de efluente sintético de industria textil aplicando tecnología avanzada de electrocoagulación. Revista Del Instituto De Investigación De La Facultad De Ingeniería Geológica, Minera, Metalúrgica Y Geográfica, 19(38), 125128. Recuperado a partir de https://revistasinvestigacion. unmsm.edu.pe/index.php/iigeo/article/view/13578

Calderón, M., \& Aramburú, V. (2018). Estudio de la electrocoagulación para controlar la turbidez y el $\mathrm{pH}$ en el tratamiento de efluentes minero metalúrgicos polimetálicos. Revista Del Instituto De Investigación De La Facultad De Ingeniería Geológica, Minera, Metalúrgica $Y$ Geográfica, 20(39), 119-122. Recuperado a partir de https:// revistasinvestigacion.unmsm.edu.pe/index.php/iigeo/ article/view/14174

Dávila J.A., Machuca F. \& Marrianga N. (2011). Treatment of vinasses by electrocoagulation-electroflotation using the Taguchi method. Electrochimica Acta. Volume 56, Issue 22, 1, September 2011, pp. 7433-7436. DOI:https://doi. org/10.1016/j.electacta.2011.07.015

Ghaitidak, D.M, \& Yadav, K.D. (2013). Characteristics and treatment of greywater-a review. Environ Sci Pollut Res. (2013) May 2013, Volume 20, Issue 5, pp 2795-2809. DOI: https://doi.org/10.1007/s11356-013-1533-0

Irdemez, S., Demircioglu, N., \& Yildiz, Y. (2006). The effects of $\mathrm{pH}$ on phosphate removal from wastewater by electrocoagulation with iron plate electrodes. Journal of Hazardous Materials Volume 137, Issue 2, pp. 1231-1235. DOI: https://doi.org/10.1016/j.jhazmat.2006.04.019

J.F.Martínez, Villafañe \& C. Montero-Ocampo (2009). Optimisation of energy consumption in arsenic electroremoval from groundwater by the Taguchi method. Separation and Purification Technology. Volume 70, Issue 3, pp. 302-305. DOI: https://doi.org/10.1016/j. seppur.2009.10.011

Kariuki, F., Kotut, K., \& Ngángá, V. (2011). The Potential of a Low Cost Technology for The Greywater Treatment. The Open Environmental Engineering Journal 4, 32-39. Kenyatta University, Department of Plant and Microbial Science. DOI: https://doi.org/10.2174/1874829501104010032

Kumar, N., \& Goel, S. (2010). Factors influencing arsenic and nitrate removal from drinking water in a continuous flow electrocoagulation (EC) process. Journal of Hazardous Materials Volume 173, Issues 1-3, pp. 528-533. DOI https://doi.org/10.1016/j.jhazmat.2009.08.117

Leal, L., Temmink, H., Zeeman, G., \& Buisman, C. (2011) Characterization and anaerobicbiodegradability of grey water, Desalination. Volume 270, Issues 1-3, pp. 111-115. DOI: https://doi.org/10.1016/j.desal.2010.11.029
Li, F., Wichmann, K., \& Otterpohl, R. (2009). Review of the technological approaches for grey water treatment and reuses. Science of The Total Environment, Volume 407, Issue 11, pp. 3439-3449.DOI: https://doi.org/10.1016/j. scitotenv.2009.02.004

N.MS Kaminaria, DR Schultza, MJJS Ponte, HA Ponte y AC Netod (2006). Heavy metals recovery from industrial wastewater using Taguchi method. Chemical Engineering Journal. Volume 126, Issues 2-3, pp. 139-146. DOI: https:// doi.org/10.1016/j.cej.2006.08.024

Roy, R. (2001). Design of experiments using the Taguchi approach: 16 steps to product and process improvement. Toronto: John Wiley \& Sons Eds. Recuperado de http://bit. ly/33ilWJx

Ulu, F., Barısçı, B., Kobya, M., \& Sillanpää, M. (2014). An evaluation on different origins of natural organic matters using various anodes by electrocoagulation. Chemosphere. Volume 125, pp. 108-114. DOI:https://doi.org/10.1016/j. chemosphere.2014.11.063

Vakil, K., Sharma, M., Bhatia, A., Kazmi, A., \& Sarkar, S. (2014). Characterization of greywater in an Indian middle-class household and investigation of physicochemical treatment using electrocoagulation. Separation and Purification Technology 130, pp. 160-166. DOI:https://doi.org/10.1016/j. seppur.2014.04.018

Vepsäläinen, M., Pulliainen, M., \& Sillanpää, M. (2012). Effect of electrochemical cell structure on natural organic matter (NOM) removal from surface water through electrocoagulation (EC). Separation and Purification Technology 99, pp. 20-27. DOI: https://doi.org/10.1016/j. seppur.2012.08.011

Yavuz, Y., Ocal, E., Koparal, A., \& O gutveren, U. (2011). Treatment of dairy industry wastewater by EC and EF processes using hybrid $\mathrm{Fe}-\mathrm{Al}$ plate electrodes. $J$ Chem Technol Biotechnol 86, pp. 964-969. DOI: https://doi. org/10.1002/jctb. 2607

Tolentino Castillo, A. J., Benites Alfaro, E., \& Cabrera Carranza, C. (2019). Aguas residuales de lavandería y su tratamiento por Oxidación fotocatalítica con dióxido de titanio (TiO2) y luz ultra violeta (UV) en Instituto Nacional de Salud del Niño, San Borja- 2017. Revista Del Instituto De Investigación De La Facultad De Ingeniería Geológica, Minera, Metalúrgica Y Geográfica, vol. 22 n 43, 2019: 3-8. Recuperado de https://revistasinvestigacion.unmsm.edu.pe/ index.php/iigeo/article/view/16680 
